\title{
IMPLEMENTACIÓN DE CAPAS SUPERIORES DE LA PIRÁMIDE DE AUTOMATIZACIÓN EN UNA PLANTA PILOTO HÍBRIDA
}

\author{
Daniel Montes ${ }^{\mathrm{a}}$, Jesús M. Zamarreño ${ }^{\mathrm{a}, \mathrm{c}}$, José Luis Pitarch ${ }^{\mathrm{b}}$, Erika Oliveira-Silva ${ }^{\mathrm{a}, \mathrm{c}}$, Cesar de Prada ${ }^{\mathrm{a}, \mathrm{c}}$ \\ danielalberto.montes.lopez@uva.es, jesusm@autom.uva.es, \\ jlpitarch@isa.upv.es, erika.oliveira@autom.uva.es,prada@autom.uva.es \\ a Departamento de Ingeniería de Sistemas y Automática, Universidad de Valladolid \\ ${ }^{\mathrm{b}}$ Instituto de Automática e Informática Industrial (ai2), Universitat Politècnica de Valencia \\ ${ }^{\mathrm{c}}$ Instituto de Procesos Sostenibles, Universidad de Valladolid
}

\begin{abstract}
Resumen
En este trabajo se presenta la implementación de un controlador predictivo basado en un modelo no lineal (NMPC) y un optimizador de consignas en tiempo real (RTO) a una planta piloto híbrida. La planta consta de un reactor CSTR, dos caudalímetros, una bomba, una válvula y cuatro sensores de temperatura. La reacción química se emula a partir de las medidas del proceso y el calor que esta generaría se aplica mediante resistencias eléctricas. Así, la única sustancia involucrada es agua, se conserva la hidrodinámica del proceso y se evitan los inconvenientes típicos del manejo de sustancias químicas. Se desarrolla y ajusta un modelo basado en medidas reales que luego es usado como base para el MPC y el RTO. Se hacen pruebas de seguimiento de consigna del controlador y optimización económica del RTO. El conjunto planta híbrida $+M P C+R T O$ conforma una plataforma flexible, a la vez que realista, para evaluar técnicas de optimización avanzada donde existen discrepancias entre planta-modelo y entre modelos.
\end{abstract}

Palabras clave: Industria 4.0, Pirámide automatización, MPC, RTO, digitalización.

\section{INTRODUCCIÓN}

En ediciones anteriores de las Jornadas de Automática se ha presentado una versión inicial del reactor híbrido del Grupo de Control y Supervisión de Procesos de la Universidad de Valladolid [1]. Desde entonces, ha habido grandes cambios tanto de hardware como de software en la planta. Se adquirió un nuevo depósito para el reactor que incorpora directamente las resistencias eléctricas. Simplificando así la planta y logrando que la dinámica se asemeja más a la de un reactor real. Por otra parte, se conectó la planta a una base de datos en tiempo real y se rediseñó la interfaz gráfica del SCADA para que tuviera cierta correspondencia con la pirámide de automatización. Se sustituyó el sistema de control en cascada por un controlador predictivo no lineal y un optimizador en tiempo real.

Las ventajas de técnicas avanzadas de optimización, detección de fallos, estimación y/o cuantificación de la incertidumbre, etc. deberían ser probadas previamente a su implementación final. Sin embargo, las plantas reales tienen una complejidad inherente que hace que la experimentación sea costosa o directamente imposible. Siempre existe el riesgo de perder producto y, además, los cambios admisibles en las variables manipuladas suelen ser muy pequeños como para obtener información dinámica valiosa. Este aspecto es todavía más crítico en el marco de la digitalización, ya que el modelado mediante aprendizaje profundo o de combinación de MPC con técnicas de aprendizaje por refuerzo requieren de una ingente cantidad de datos informativos sobre el proceso. Si dichos datos no pueden ser obtenidos mediante experimentación directa, la mayoría de propuestas en la literatura asumen que se dispone de un modelo de simulación detallado del proceso como alternativa para realizar el aprendizaje. No obstante, es difícil que un modelo llegue a reflejar toda la realidad y problemas de operación de una planta real.

Las plantas piloto buscan asemejarse a los procesos reales pero suelen tener poca instrumentación y son de muy pequeña escala. En éstas normalmente están involucradas las mismas sustancias que en los procesos reales. Así, se deben tener consideraciones de adquisición de sustancias químicas, disposición de las mismas, medidas de seguridad, etc. A medio camino entre las plantas reales y las piloto se encuentran las plantas piloto híbridas. De esta forma, parte del proceso es simulada mientras que las características hidrodinámicas se mantienen fieles a la realidad [2].

La tradicional pirámide de automatización, mostrada en la figura 1 , recoge las tecnologías asociadas a la automatización industrial y a la gestión de procesos productivos. Está organizada jerárquicamente en diferentes niveles: proceso, dispositivos, PLC/SCADA, MES (Manufacturing Execution System) y ERP (Enterprise Resource Planning). Es importante destacar que no se dede avanzar a niveles superiores si los inferiores no funcionan adecuadamente. Esta separación entre capas es mucho más que un concepto y tiene implicaciones tanto en la organización de las empresas como en los flujos de información de los procesos. Las tecnologías relacionadas con el control predictivo se pueden situar entre el nivel de PLC/SCADA y el de MES. Por su parte, las tecnologías asociadas con la 
optimización en tiempo real se emplazan en la capa de MES. Así, este trabajo servirá como demostración de la implementación de estrategias de las capas superiores de la pirámide.

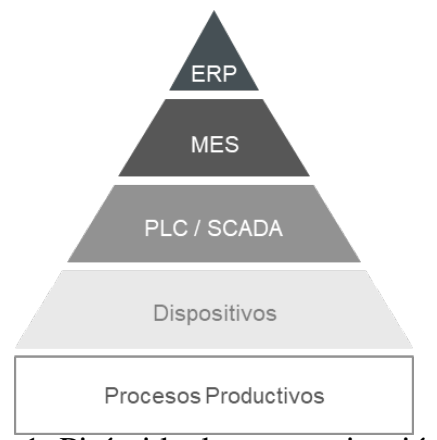

Figura 1: Pirámide de automatización [3].

En la sección 2 se explica en detalle la configuración física de la planta. La sección 3 describe su arquitectura de software. En la sección 4 se desarrolla y ajusta un modelo. Luego, en la sección 5 se formulan un controlador predictivo no lineal y un optimizador en tiempo real. Finalmente, la sección 6 muestra las pruebas llevadas a cabo con estas herramientas de control avanzado. El artículo se cierra con las conclusiones y propuestas de trabajo futuro en la sección 7.

\section{DESCRIPCIÓN DE LA PLANTA}

La principal característica de la planta es que, aunque tiene todos los elementos de un reactor químico, la reacción está simulada y la única sustancia involucrada es agua. Una simulación de la reacción recibe medidas en tiempo real del proceso para calcular el calor que generaría la reacción, este luego se aplica mediante resistencias eléctricas.

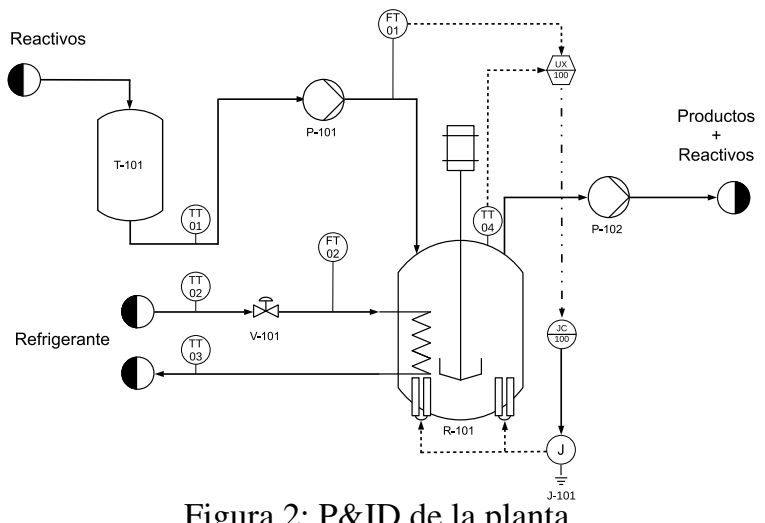

Figura 2: P\&ID de la planta.

En la figura 2 se muestra un esquema de la planta. Esta cuenta con cuatro sensores de temperatura (TT-01 a 04) y dos caudalímetros (FT-01 y 02). Los elementos finales de control son la bomba peristáltica de reactivos (P-101) y la válvula de refrigerante (V-101). La bomba P-102 tiene una velocidad fija y su única función es simular que los reactivos salen del reactor por desbordamiento.

La simulación de la reacción química está embebida en un servidor OPC-DA generado con EcosimPro [4]. En el trabajo original de Marcos et al. solo había una reacción, A que produce $\mathrm{B}$ [1]. Ahora se ha cambiado para incluir las reacciones de van de Vusse, conocidas por su alta no linealidad [5].

$$
\begin{gathered}
\mathrm{A} \stackrel{\mathrm{k}_{1}}{\longrightarrow} \mathrm{B} \stackrel{\mathrm{k}_{2}}{\longrightarrow} \mathrm{C} \\
2 \mathrm{~A} \stackrel{\mathrm{k}_{3}}{\longrightarrow} \mathrm{D}
\end{gathered}
$$

Las ecuaciones que están programadas dentro del bloque de simulación son iguales a las que se mostrarán en la sección 4.1. Una vez se ha calculado el calor que generarían las reacciones, este se envía como una señal a la unidad de amplificación de las resistencias eléctricas. De esta forma, se consigue emular la dinámica de un reactor CSTR.

\section{ARQUITECTURA SOFTWARE}

La planta piloto se ha dotado de una arquitectura que se asemeja a una instalación industrial. Por una parte, se extendió el desarrollo de Marcos et al. [1] del SCADA en el software Wonderware InTouch [6]. También se conectó la planta a una base de datos en tiempo real, PI System de OSIsoft [7], para tener acceso al histórico de todos los datos del proceso. Los controladores PID de la planta y la tarjeta de adquisición de datos se comunican mediante OPC-DA [8]. Sigue siendo ampliamente utilizado en la industria aunque su sucesor, OPC-UA, fue publicado hace más de una decada. El nuevo estándar de OPC-UA pretende mejorar las carencias de su predecesor: no depende de COM y, por tanto, es multiplataforma; tiene en cuenta aspectos de ciberseguridad; se puede paralelizar en múltiples CPUs; tiene soporte de redundancia, etc. [9]. En nuestro trabajo se usa este último estándar para los módulos de control avanzado y optimización en tiempo real.

\subsection{SCADA}

El SCADA original fue desarrollado por Marcos et al. [1] usando Wonderware InTouch [6]. Este recogía toda la información del proceso y permitía actuar sobre él. Además, tenía implementados dos lazos de control en cascada: uno para la temperatura del reactor y otro para la conversión. En este trabajo se ha reorganizado el SCADA para que reflejara de forma visual la pirámide de automatización. En la figura 3 se puede ver la ventana principal de la interfaz hombre-maquina (HMI) del SCADA de la planta. De especial interés es la pirámide en la esquina superior derecha. Esta permite navegar entre las diferentes ventanas por niveles de la pirámide. En cada una de las ventanas se pueden configurar los módulos a los que corresponden y visualizar algunas variables de interés. 


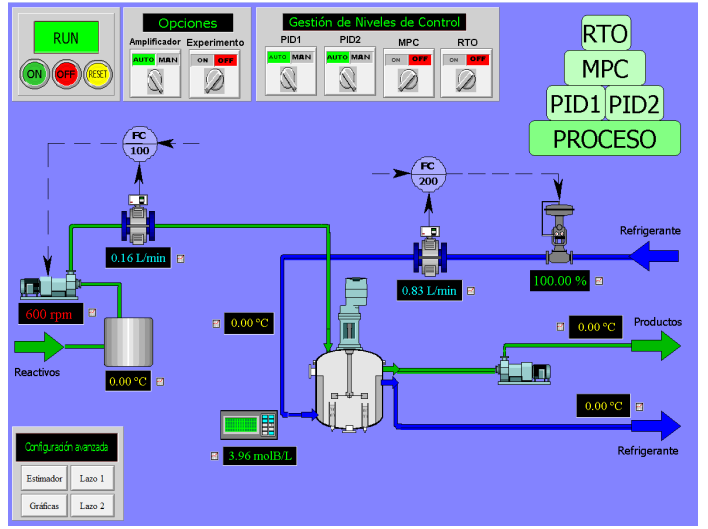

Figura 3: Ventana principal del HMI.

\subsection{BASE DE DATOS EN TIEMPO REAL}

Las bases de datos en tiempo real permiten gestionar la gran cantidad de datos que se generan en los procesos en cada periodo de muestreo. Con ellas se busca facilitar el acceso a la información y así basar las decisiones corporativas en los datos. Aunque el concepto es relativamente sencillo, una buena base de datos debe tener capacidades de comunicación con prácticamente cualquier protocolo que se use en la industria (hay cientos). Además, también deben disponer de herramientas que permitan analizar y visualizar la información en tiempo real para descubrir patrones de interés. Todas estas características están incluidas en el PI System de OSIsoft [7], software que se usa de forma generalizada en la industria de procesos y energía.

Con el fin de que la planta se asemeje aún más a una instalación industrial, se conectó la misma a una instancia ya creada del PI System [10]. Así también se emula la estructura de la información típica de una empresa. Específicamente, se conectaron la tarjeta de adquisición de datos, los controladores PID, el SCADA (OPC-DA) y los bloques de control avanzado y optimización en tiempo real (OPC-UA). La figura 4 muestra la configuración de las variables en el servidor PI Asset Framework (PI AF) del PI System.

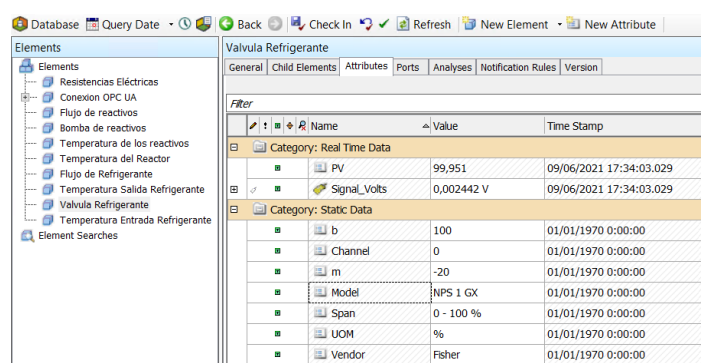

Figura 4: Estructura en el servidor PI AF.

\section{MODELADO Y AJUSTE}

\subsection{Modelo}

Se debe desarrollar un modelo que sirva como base para las estrategias de control avanzado y optimización que se implementen en la planta. Se supone que las propiedades físicas del agua permanecen constantes y que el reactor está perfectamente agitado. Así, se usa el modelo típico de un reactor CSTR. También se supone que las reacciones químicas son conocidas, pero no así sus parámetros.

Se formulan los balances de materia teniendo en cuenta que solo entra al reactor el reactivo A.

$$
\begin{aligned}
V \frac{d C_{A}}{d t} & =q\left(C_{A 0}-C_{A}\right)+V\left(-r_{1}-2 r_{3}\right) \\
V \frac{d C_{B}}{d t} & =-q C_{B}+V\left(r_{1}-r_{2}\right) \\
V \frac{d C_{C}}{d t} & =-q C_{C}+r_{2} V \\
V \frac{d C_{D}}{d t} & =-q C_{D}+r_{3} V
\end{aligned}
$$

Donde $C_{i}$ son las concentraciones de cada una de las especies $\left(\frac{\mathrm{mol}}{\mathrm{L}}\right), V$ el volumen del reactor $(\mathrm{L}), q$ es el caudal de reactivos $\left(\frac{\mathrm{L}}{\min }\right)$ y $r_{j}$ las velocidades de reacción $\left(\frac{\mathrm{mol}}{\mathrm{Lmin}}\right)$.

Los balances de energía representan la parte del proceso que no es simulada, es decir, el proceso real. Se incluyen términos de generación de energía y de intercambio de calor. El serpentín también se modela como si su temperatura fuera homogénea.

$$
\begin{gathered}
\rho C_{p} V \frac{d T}{d t}=q \rho C_{p}\left(T_{0}-T\right)-U A\left(T-T_{c}\right)+Q_{r x n} \\
\rho C_{p} V_{c} \frac{d T_{c}}{d t}=F r \rho C_{p}\left(T_{c 0}-T_{c}\right)+U A\left(T-T_{c}\right)
\end{gathered}
$$

Donde $U A$ es el coeficiente global de transferencia de calor $\left(\frac{\mathrm{kJ}}{\min ^{\circ} \mathrm{C}}\right), Q_{r x n}$ es el calor generado por las reacciones químicas ( $\left.\frac{\mathrm{kJ}}{\mathrm{min}}\right)$ y $F r$ es el caudal de refrigerante ( $\left.\frac{\mathrm{L}}{\min }\right)$. Además, $T_{0}$ y $T_{c 0}$ son las temperaturas del reactor y el serpentín a la entrada, mientras que $T$ y $T_{c}$ son las temperaturas a la salida.

Como se mencionó anteriormente, los parámetros de las reacciones se suponen desconocidos. Así, se deben encontrar valores para los mismos de forma tal que el modelo se ajuste adecuadamente al proceso. Las ecuaciones cinéticas son como sigue:

$$
\begin{aligned}
r_{1} & =k_{10} e^{-\frac{E_{1}}{R T}} C_{A} \\
r_{2} & =k_{20} e^{-\frac{E_{2}}{R T}} C_{B} \\
r_{3} & =k_{30} e^{-\frac{E_{3}}{R T}} C_{A}^{2} \\
Q_{r x n} & =-V \sum_{j=1}^{3} r_{j} \Delta H_{r x n, j} \\
U A & =\alpha F r^{0.8}
\end{aligned}
$$

Donde $k_{j 0}$ son las constantes pre-exponenciales de las velocidades cinéticas $\left(\frac{1}{\mathrm{~min}}\right), E_{j}$ las energías de activación de las reacciones $\left(\frac{\mathrm{kJ}}{\mathrm{mol}}\right)$ y $\Delta H_{r x n, j}$ las entalpías de 
las reacciones $\left(\frac{\mathrm{kJ}}{\mathrm{mol}}\right) . \alpha$ es un parámetro que engloba a otros como las propiedades del agua, el diámetro del serpentín, etc.

En la tabla 1 se muestran los valores de los parámetros conocidos del modelo.

Tabla 1: Parámetros conocidos del modelo.

\begin{tabular}{clc}
\hline Parám. & Descripción & Valor \\
\hline$V$ & Volumen del reactor & $11.5 \mathrm{~L}$ \\
$V_{c}$ & Volumen del serpentín & $1 \mathrm{~L}$ \\
$\rho$ & Densidad del agua & $1 \frac{\mathrm{kg}}{\mathrm{L}}$ \\
$C_{p}$ & Capacidad calorífica & $4.18 \frac{\mathrm{kJ}}{\mathrm{kg}^{\circ} \mathrm{C}}$ \\
$C_{A 0}$ & Conc. entrada de $\mathrm{A}$ & $5 \frac{\mathrm{mol}}{\mathrm{L}}$ \\
\hline
\end{tabular}

Las ecuaciones (1)-(11) serán lo que se denomine de ahora en adelante como el modelo de la planta. Por otra parte, conviene diferenciar el modelo de la planta de lo que está programado dentro del bloque que emula la reacción química, ecuaciones (1)-(4) y (7)-(10). A pesar de ser exactamente las mismas ecuaciones, la emulación de las reacciones no está afectada únicamente por estos balances de materia sino también por las dinámicas de la unidad de amplificación de potencia y de las resistencias eléctricas. Esto último no se tiene en cuenta para el modelo de la planta y, por tanto, hay errores estructurales que impedirán que el modelo represente perfectamente la dinámica de la planta.

\subsection{AJUSTE}

Se llevó a cabo un experimento para recolectar datos dinámicos de la planta y ajustar el modelo a los mismos. Se dieron valores diferentes a la velocidad de la bomba de reactivos y a la apertura de la válvula refrigerante. Se intentó explorar todas las zonas posibles de operación de la planta y que los cambios no estuvieran correlacionados. En la figura 5 se muestra la evolución de los caudales de reactivos y refrigerante durante el experimento.

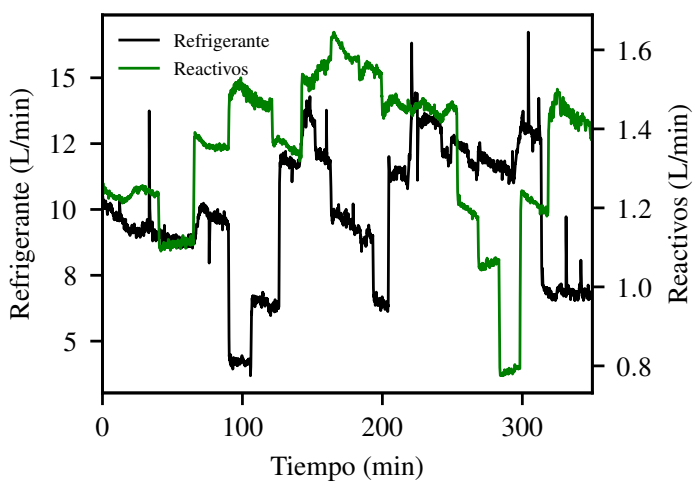

Figura 5: Planificación del experimento.

Una vez recolectados los datos, se formula un problema de optimización dinámica como se muestra en la ecuación (12). Las restricciones corresponderían a las ecuaciones del modelo (1)-(11). Para la función de costo se usó una fair function en lugar de mínimos cuadrados. Esto limita la penalización ante errores gruesos, i.e. grandes diferencias entre modelo y medida de algún sensor (descalibraciones, etc.), evitando así distorsionar el ajuste a otras variables cuyas medidas son más fiables. [11].

$$
\begin{array}{cl}
\min _{\substack{k_{i}, E_{i}, \Delta H_{r x n, i}, \alpha}} & c^{2} \sum_{i=1}^{4} \gamma_{i}\left[\frac{\left|\varepsilon_{i}\right|}{c}-\ln \left(1+\frac{\left|\varepsilon_{i}\right|}{c}\right)\right] \\
\text { s.t. } & \text { Ecuaciones (1)-(11), } \\
& \varepsilon_{i}=\frac{y_{m, i}-y_{p, i}}{\widehat{y}_{p, i}} \quad i=1, \ldots, 4
\end{array}
$$

Donde $c$ es un hiperparámetro con valor de seis y $\gamma_{i}$ y $\varepsilon_{i}$ son los pesos asociados y los errores normalizados respecto a cada uno de los estados medidos. $y_{m, i}$ son las predicciones del modelo, $y_{p, i}$ las medidas reales del proceso y $\widehat{y}_{p, i}$ la media de cada una de las medidas.

Para resolver el problema de optimización dinámica (12) se siguió un enfoque secuencial, usando EcosimPro [4] para evaluar la función de costo y SNOPT [12] para calcular nuevos valores de los parámetros. La optimización secuencial consiste en usar un simulador para resolver las ecuaciones diferenciales y así evaluar la función de costo y las restricciones [13]. Estas son enviadas a un optimizador que se encarga de calcular nuevos valores para las variables de decisión y se repite el proceso. La iteración se detiene cuando se ha cumplido el número máximo de iteraciones o la tolerancia especificada en el optimizador. La figura 6 muestra un esquema simplificado de la optimización secuencial.

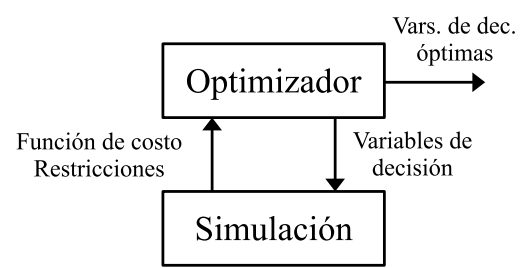

Figura 6: Optimización secuencial.

Del experimento de 350 minutos de duración, se usaron 250 para ajustar el modelo y 100 para validarlo. Las figuras 7, 8 y 9 muestran el resultado gráfico del ajuste. La tabla 2 muestra los valores de los parámetros.

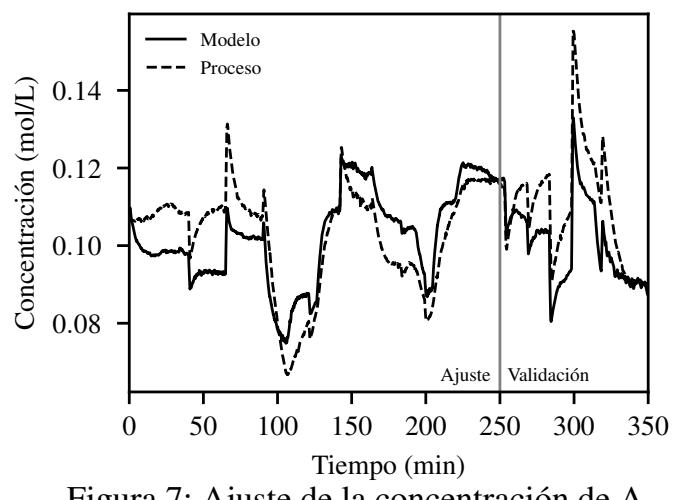

Figura 7: Ajuste de la concentración de A. 


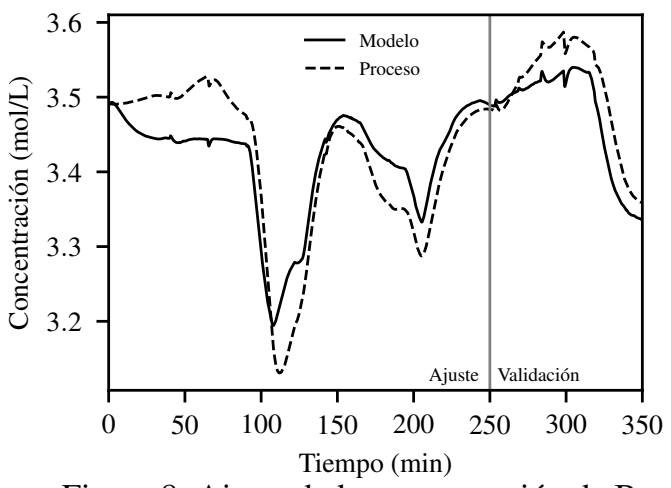

Figura 8: Ajuste de la concentración de B.

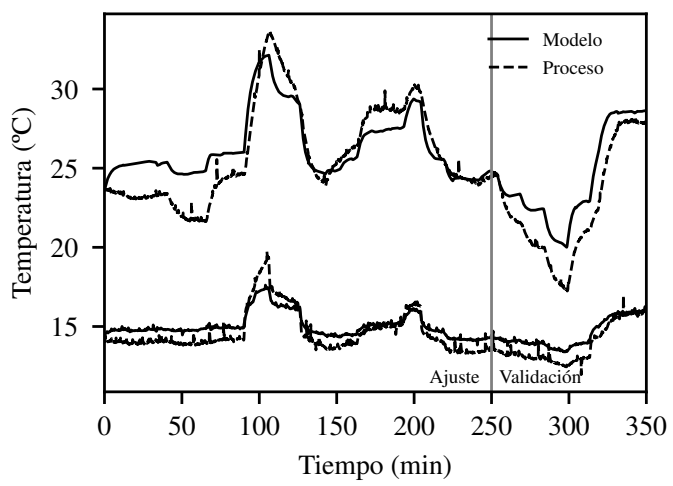

Figura 9: Ajuste de las temperaturas.

Se puede apreciar que el ajuste del modelo, aunque no perfecto, es bueno. Se capturan adecuadamente los transitorios del proceso, pero hay cierto error de estado estacionario. Este error debería ser capaz de remediarlo un estimador de estados de horizonte móvil como se mostrará en la sección 5.1.2.

Tabla 2: Parámetros ajustados del modelo

\begin{tabular}{|c|c|c|}
\hline Parám. & Descripción & Valor \\
\hline$k_{10}$ & Cte. pre-exp. $1\left(\mathrm{x} 10^{9}\right)$ & $6.14 \frac{1}{\min }$ \\
\hline$k_{20}$ & Cte. pre-exp. $2\left(\times 10^{10}\right)$ & $1.71 \frac{1}{\min }$ \\
\hline$k_{30}$ & Cte. pre-exp. $3\left(\times 10^{11}\right)$ & $8.28 \frac{1}{\operatorname{minmol}}$ \\
\hline$E_{1}$ & Energía de activación 1 & $52.1 \frac{\mathrm{kJ}}{\mathrm{mol}}$ \\
\hline$E_{2}$ & Energía de activación 2 & $73.2 \frac{\mathrm{mol}}{\mathrm{mol}}$ \\
\hline$E_{3}$ & Energía de activación 3 & $64.9 \frac{\mathrm{kJ}}{\mathrm{mol}}$ \\
\hline$\Delta H_{r x n, 1}$ & Entalpía de 1 & $-19.87 \frac{\mathrm{mJ}}{\mathrm{mol}}$ \\
\hline$\Delta H_{r x n, 2}$ & Entalpía de 2 & $-3.16 \frac{\mathrm{kJ}}{\mathrm{mol}}$ \\
\hline$\Delta H_{r x n, 3}$ & Entalpía de 3 & $-77.42 \frac{\mathrm{kJ}}{\mathrm{mol}}$ \\
\hline$\alpha$ & Transferencia de calor & $1.62 \frac{\mathrm{kJ}}{\min ^{\circ} \mathrm{C}}$ \\
\hline
\end{tabular}

\section{CONTROL AVANZADO Y RTO}

\subsection{CONTROLADOR PREDICTIVO}

\subsubsection{Controlador}

La función de coste tiene términos de seguimiento de consigna para la temperatura y la concentración de B. Además, se consideran términos de penalización de cambios en las variables manipuladas. Las acciones de control se discretizan de forma constante entre cada periodo de muestreo. El resultado del MPC se envía a la planta como consignas a los controladores PID de caudal. Por último, se añaden restricciones de cotas inferiores y superiores para las variables controladas y las manipuladas. Los parámetros del controlador se muestran en la tabla 3 .

$$
\begin{aligned}
& \min _{\substack{u_{1}(t), u_{2}(t)}} \sum_{k=1}^{2} \sum_{i=0}^{N} \gamma_{k}\left[\frac{y_{k}(t+i)-w_{k}(t+i)}{y_{k, \text { upp }}-y_{k, \text { low }}}\right]^{2} \\
& +\sum_{j=1}^{2} \sum_{i=0}^{N u} \beta_{j}\left[\frac{\Delta u_{j}(t+i)}{u_{j, u p p}-u_{j, l o w}}\right]^{2} \\
& y_{k, \text { low }} \leq y_{k} \leq y_{k, u p p} \text {, } \\
& k=1, \ldots, 4, \\
& u_{j, \text { low }} \leq u_{j} \leq u_{j, u p p} \text {, } \\
& j=1,2
\end{aligned}
$$

Donde $u_{1}(t)$ y $u_{2}(t)$ son los vectores de variables manipuladas a lo largo del horizonte de control, $y_{k}(t)$ y $w_{k}(t)$ son las variables controladas y su consigna a lo largo del horizonte de predicción, $y_{k, u p p}$ y $y_{k, l o w}$ son los límites superiores e inferiores de las variables controladas y $u_{k, \text { upp }}$ y $u_{k, l o w}$ son los límites superiores e inferiores de las variables manipuladas.

Tabla 3: Parámetros del controlador.

\begin{tabular}{clc}
\hline Parám. & Descripción & Valor \\
\hline$N u$ & Horizonte de control & 3 \\
$N$ & Horizonte de predicción & 120 \\
$\gamma_{k}$ & Peso & 1.0 \\
$\beta_{j}$ & Penalizaciones & 2.0 \\
$\Delta t$ & Periodo de muestreo & $0.5 \mathrm{~min}$ \\
$u_{1, l o w}$ & Caudal min. reactivos & $0.3 \frac{\mathrm{L}}{\mathrm{min}}$ \\
$u_{1, \text { upp }}$ & Caudal max. reactivos & $1.2 \frac{\mathrm{L}}{\mathrm{min}}$ \\
$u_{2, l o w}$ & Caudal min. refrigerante & $6 \frac{\mathrm{L}}{\mathrm{min}}$ \\
$u_{2, \text { upp }}$ & Caudal max. refrigerante & $15 \frac{\mathrm{L}}{\mathrm{min}}$ \\
\hline
\end{tabular}

\subsubsection{Estimador de estados}

Aunque para el ajuste del modelo se consideró que se medía la especie A, para la operación normal de la planta se considera que esta medida no está disponible. Se formuló un estimador de estados de horizonte móvil (MHE) que estime esta concentración no medida. El MHE considera un horizonte móvil de $N_{E}$ medidas anteriores y es ejecutado antes de cada llamada al controlador, estimando los estados al inicio del horizonte y las perturbaciones. Estimar las perturbaciones dota al controlador de acción integral, eliminando posibles errores de estado estacionario [14]. Luego se integra el modelo desde el inicio del horizonte hasta el periodo de muestreo actual para enviar los valores actualizados al controlador.

La función de costo que se presenta en la ecuación (14) está compuesta por tres términos: penalización de la diferencia entre los estados y la variables medidas; penalización de desviación con las estimaciones iniciales 
anteriores y penalización de las perturbaciones estimadas. A diferencia de los problemas de optimización de ajuste de parámetros y MPC, el modelo del estimador de estados tiene una ligera modificación. Se incorporan perturbaciones aditivas $\left(d_{1}, \ldots, d_{4}\right)$ a las ecuaciones (1), (2), (5) y (6) respectivamente. Estos términos también se incluyen en el modelo del MPC. En la ecuación (15) se muestra un ejemplo de como se incorporan en el modelo. Una vez estimadas por el MHE, el controlador considera las perturbaciones $\left(d_{1}, \ldots, d_{4}\right)$ como constantes durante todo el horizonte de predicción.

$$
\begin{aligned}
\min _{\substack{y_{k}\left(t-N_{E}\right), d_{k}}} & \beta_{m} \sum_{k=1}^{4} \sum_{i=0}^{N_{E}}\left[\gamma_{k}\left(\frac{y_{k}(t-i)}{y_{k, \text { real }}(t-i)}-1\right)^{2}\right] \\
+ & \beta_{\text {ant }} \sum_{k=1}^{4} \gamma_{k}\left(y_{k}\left(t-N_{E}\right)-y_{k, \text { ant }}\left(t-N_{E}\right)\right)^{2} \\
& +\beta_{d} \sum_{k=1}^{4} d_{k}^{2}
\end{aligned}
$$

s.t. Ecuaciones (1)-(11),

$$
\begin{array}{ll}
y_{k, \text { low }} \leq y_{k} \leq y_{k, \text { upp }}, & k=1, \ldots, 4, \\
d_{k, \text { low }} \leq d_{k} \leq d_{k, \text { upp }}, & k=1, \ldots, 4
\end{array}
$$

Donde $\beta_{m}, \beta_{a n t}, \beta_{d}$ son los pesos relativos asociados con las medidas de los estados, las estimaciones anteriores y las perturbaciones respectivamente. Así mismo, $\gamma_{k}$ son los pesos relativos al ajuste a las medidas de cada uno de los estados. Finalmente, $N_{E}$ es el número de periodos de muestreo considerados (longitud del horizonte).

$$
V \frac{d C_{A}}{d t}=q\left(C_{A 0}-C_{A}\right)+V\left(-r_{1}-2 r_{3}\right)+d_{1}
$$

Tanto el controlador como el MHE (13) fueron programados en EcosimPro [4], usando IDAS para la integración y SNOPT [12] para la optimización (siguiendo un enfoque secuencial). El coste computacional es bajo, aproximadamente medio segundo para resolver ambos problemas. Estos se envolvieron dentro de un mismo servidor OPC-UA que les permita interactuar con el SCADA de la planta fácilmente. El servidor del controlador es llamado en cada periodo de muestreo y envía nuevos valores para las consignas de caudal de los controladores PID.

\subsection{OPTIMIZACIÓN EN TIEMPO REAL}

Para formular el optimizador en tiempo real se usa el modelo ya desarrollado pero en estado estacionario. El objetivo es maximizar el beneficio económico de la planta. No se tienen en cuenta las perturbaciones estimadas en el MHE. Se asume que los transitorios tienen poca relevancia en la economía del proceso por ser previsiblemente cortos. En la función de costo del problema de optimización (16) se tiene en cuenta: lo que se produce de la sustancia de interés $B$, lo que se consume de reactivos y lo que se usa de refrigerante. Así, las variables de decisión son la temperatura del reactor y la concentración de B. Estas se envían al controlador como puntos de consignas en cada llamada al RTO.

$$
\begin{array}{cl}
\max _{T, C_{B}} & q\left(p_{B} C_{B}-p_{A} C_{A 0}\right)-p_{\text {ref }} F r \\
\text { s.t. } & \text { Ecuaciones }(1)-(11), \\
& y_{k, \text { low }} \leq y_{k} \leq y_{k, \text { upp }}, k=1, \ldots, 4, \\
& u_{j, \text { low }} \leq u_{j} \leq u_{j, \text { upp }}, j=1,2
\end{array}
$$

Al igual que el MPC y el MHE, el RTO se programó en EcosimPro [4]. En este caso no fue necesario usar un integrador, pero sí un optimizador (SNOPT [12]). El RTO se envolvió con una capa de OPC-UA para facilitar su integración con la planta y el resto de módulos. Los precios de las especies involucradas en la función de costo se establecieron en $0.2,18$ y $3 € / \mathrm{mol}$ para $\mathrm{A}$, B y Fr respectivamente.

\section{PRUEBAS DE MPC Y RTO}

\subsection{Prueba del controlador predictivo}

Se partió desde un estado estacionario y luego se activó el MPC. Las consignas probadas fueron elegidas arbitrariamente. Tras unos minutos de recolección de datos para el estimador de estados, el controlador empieza a calcular nuevas acciones de control. Luego, alrededor de $t=45 \mathrm{~min}$, se cambian simultáneamente las dos consignas. En las figuras 10 y 11 se muestra la evolución de las variables controladas y manipuladas. La escala de los ejes de la figura 11 refleja los límites de las variables manipuladas.

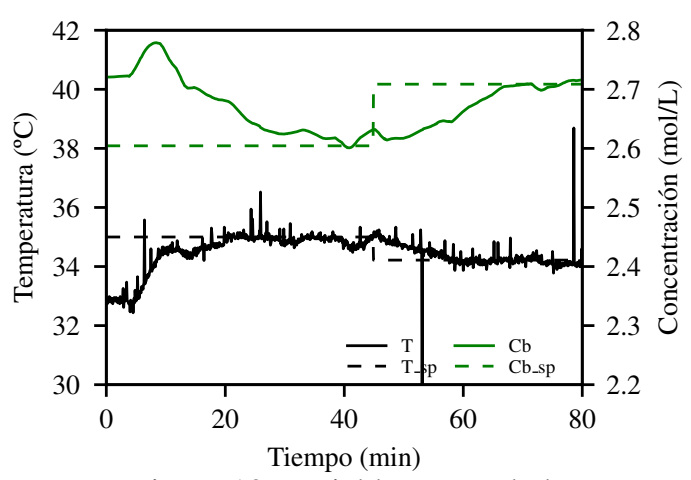

Figura 10: Variables controladas.

El controlador es capaz de llevar al proceso a las dos condiciones de operación especificadas pero hay oscilaciones en las variables manipuladas. Aumentar la penalización a los cambios $\left(\beta_{j}\right)$ ayudaría a disminuir estas oscilaciones. No obstante, creemos que estas se deben principalmente al ruido, ya que la implementación actual no considera el filtrado de las señales. En cualquier caso, el rendimiento del controlador es satisfactorio. 


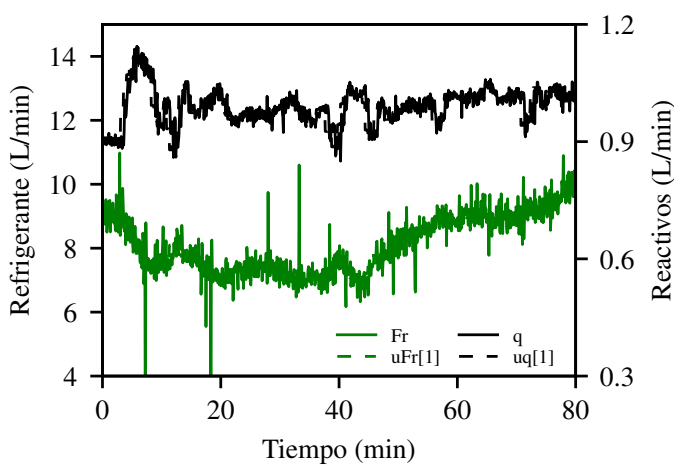

Figura 11: Variables manipuladas.

\subsection{Prueba del optimizador en tiempo real}

Para probar el RTO, se partió de un estado estacionario al igual que para la prueba del MPC. En las figuras 12,13 y 14 se muestra la evolución de las variables controladas, de las variables manipuladas y de la economía del proceso. Tras recolectar datos para el estimador de estados, el MPC empieza a calcular acciones de control para llegar a las consignas que le llegan desde el RTO.

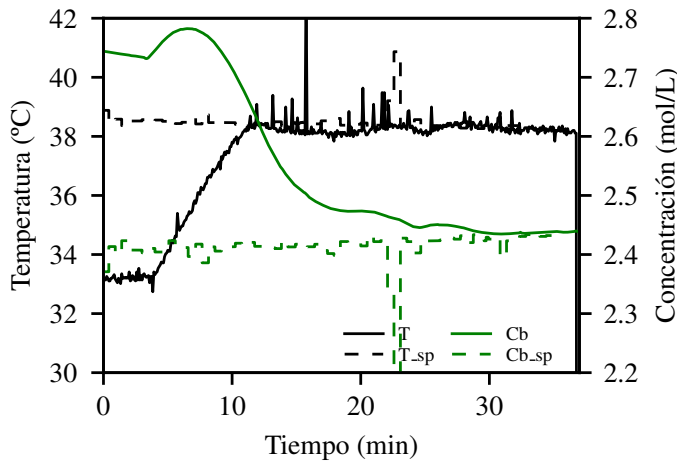

Figura 12: Variables controladas.

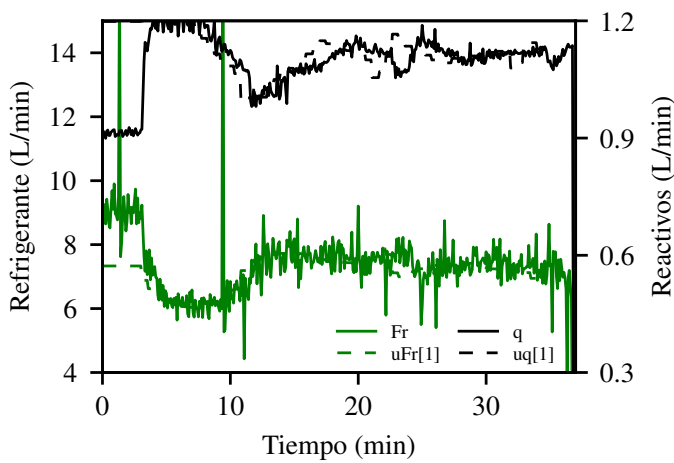

Figura 13: Variables manipuladas.

Se puede apreciar que los puntos de operación óptimos calculados por el RTO tienden a llevar a las variables manipuladas cerca de sus límites. El caudal de reactivos está cerca del límite superior $\left(1.2 \frac{\mathrm{L}}{\min }\right)$ y el de refrigerante cerca del límite inferior $\left(6 \frac{\mathrm{L}}{\mathrm{min}}\right)$. El comportamiento del MPC es adecuado ya que es capaz de llevar al proceso a la zona indicada por el RTO sin error de estado estacionario. Por otra parte, en la figura 14

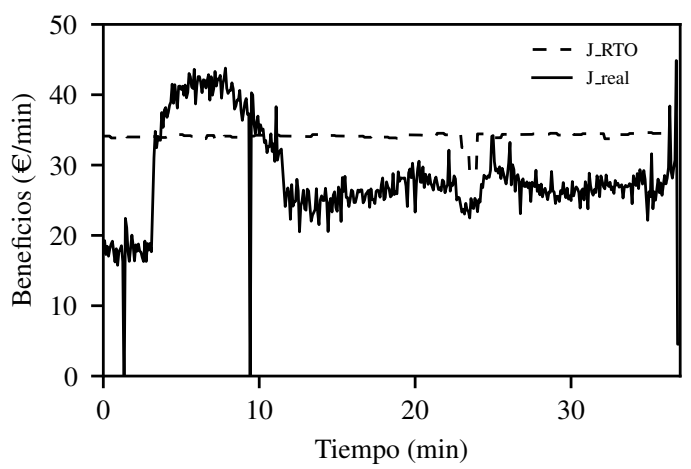

Figura 14: Economía del proceso.

se muestra el valor de la función de costo que calcula el RTO y el valor que hay realmente en la planta. Si el modelo fuera perfecto, estos valores tendrían que coincidir. Así, aunque el RTO logra llevar a la planta a una mejor zona de operación, en términos económicos, comparada con el estacionario inicial, esta zona probablemente no es el punto de operación óptimo de la planta.

\section{CONCLUSIONES Y TRABAJO FUTURO}

Se ha usado una planta piloto híbrida como eje central del trabajo. Se desarrolló y ajustó un modelo para la misma, que luego sirvió como base para formular un MPC y un RTO. El controlador predictivo tiene incorporado un MHE para eliminar el error de estado estacionario. Por su parte, el optimizador en tiempo real usa el modelo de la planta en estado estacionario para calcular el punto de operación que maximiza el beneficio económico. Ambos módulos se conectan directamente al SCADA de la planta mediante el protócolo de comunicación OPC-UA. Las pruebas llevadas a cabo demostraron el correcto funcionamiento tanto del MPC como el RTO. Así, se comprueba la utilidad que tienen este tipo de plantas para probar estrategias de control u optimización avanzadas antes de su implementación final en un proceso industrial.

Las características de la plataforma realizada alrededor de la planta (no linealidad, flexibilidad, software industrial) la hacen ideal para probar nuevos algoritmos de control y optimización. Como trabajo futuro, se desea probar un controlador predictivo económico con la técnica de adaptación de modificadores (MA). Esta técnica consiste en modificar la función de costo para que el óptimo del modelo sea igual al óptimo del proceso [15]. De esta forma, se lograría llegar al óptimo real de la planta y no al del modelo como ocurrió en la prueba del RTO. Por otra parte, sería interesante implementar la capa superior de la pirámide que corresponde a la planificación de la producción (ERP). Para esto, será necesario expandir el proceso interconectando varias plantas piloto que interactúen entre sí y desarrollar un modelo que las coordine. 


\section{Agradecimientos}

Los autores desean agradecer al Ministerio de Ciencia Innovación y Universidades y a la Agencia Estatal de Investigación por su financiación mediante el proyecto PGC2018-099312-B-C31. Daniel Montes ha sido financiado con cargo a la convocatoria 2020 de contratos predoctorales de la Universidad de Valladolid, cofinanciada por el Banco Santander. El segundo, cuarto y quinto autor agradecen el apoyo de la Junta de Castilla y León y EU/FEDER (CLU 2017-09).

\section{English summary}

\section{IMPLEMENTATION OF UPPER LA- YERS OF THE AUTOMATION PYRA- MID IN A HYBRID PILOT PLANT}

\begin{abstract}
This work presents the implementation of a real-time optimization (RTO) and a nonlinear model predictive controller (NMPC) to a hybrid pilot plant. The plant consists of a Van de Vusse CSTR, two flowmeters, a pump, a valve, and four temperature sensors. In order to maintain the hydrodynamics and avoid typical problems with chemical substances, all the reactions are simulated. So, the only substance involved in the process is water. A calculation block simulates the chemical reaction using a model and real-time process measurements, calculating the heat generated by the reaction. This heat is applied to the reactor using two heating coils. Another model for the RTO and NMPC layers is formulated, and the parameters are adjusted using experimental measurements. Then, two experiments are carried out: trajectory tracking for the MPC, and economic optimization for the RTO. The present paper shows the advantages of using hybrid pilot plants for the study and research of advanced control strategies.
\end{abstract}

Keywords: Industry 4.0, Automation Pyramid, MPC, RTO, digitalization.

\section{Referencias}

[1] M. P. Marcos, C. de Prada y J. L. Pitarch, «Desarrollo e implementación de un sistema de control en una planta piloto híbrida», en XXXVIII Jornadas de Automática, Universidad de Oviedo, 2017, págs. 479-485.

[2] L. Bergh, «A Hybrid Approach to Empirically Test Process Monitoring, Diagnosis and Control Strategies», Advances in Intelligent and Soft Computing, vol. 126, págs. 215-222, 2012.
[3] ISA España. «MES: Pasado, presente y mucho futuro». (2021), dirección: https : / / isaspain . org / mes - pasado - presente - y mucho-futuro/ (visitado 08-06-2021).

[4] Empresarios Agrupados. «EcosimPro». (2021), dirección: https : / / www . ecosimpro . com/ (visitado 05-06-2021).

[5] J. G. van de Vusse, «Plug-flow type reactor versus tank reactor», Chemical Engineering Science, vol. 19, n. ${ }^{\circ}$ 12, págs. 994-996, 1964.

[6] Wonderware Spain. «Wonderware InTouch». (2021), dirección: https : //www . wonderware . es / hmi - scada / intouch/ (visitado 05-06-2021).

[7] OSIsoft. «PI System». (2021), dirección: https : //www . osisoft.es/ (visitado 07-06-2021).

[8] J. M. Zamarreño, Acceso a datos mediante OPC. Santiago de Compostela: Editorial Andavira SL, 2010, ISBN: 978-84-8408-565-2.

[9] OPC Foundation. «OPC Unified Architecture». (2021), dirección: https : //opcfoundation . org / about / opc-technologies / opc-ua/ (visitado 08-06-2021).

[10] J. M. Zamarreño, C. Pablos, A. Merino, L. F. Acebes y C. de Prada, «Infraestructura para explotación de datos de un simulador azucarero», en XXXVIII Jornadas de Automática, Universidad de Oviedo, 2017, págs. 197-202.

[11] N. Arora y L. T. Biegler, «Redescending estimators for data reconciliation and parameter estimation», Computers and Chemical Engineering, vol. 25, n. ${ }^{\circ}$ 11-12, págs. 1585-1599, 2001.

[12] P. E. Gill, W. Murray y M. A. Saunders, «SNOPT: An SQP algorithm for large-scale constrained optimization», SIAM Rev., vol. 47, págs. 99-131, 2005.

[13] L. T. Biegler, Nonlinear Programming: Concepts, Algorithms and Applications to Chemical Processes. 2010.

[14] M. Vaccari y G. Pannocchia, «Implementation of an economic MPC with robustly optimal steadystate behavior», IFAC-PapersOnLine, vol. 51, n. ${ }^{\circ}$ 20, págs. 92-97, 2018.

[15] E. Oliveira-Silva, C. de Prada y D. Navia, «Dynamic optimization integrating modifier adaptation using transient measurements», Computers and Chemical Engineering, vol. 149, 2021.

(C) 2021 by the authors. Submitted for possible open access publication under the terms and conditions of the Creative Commons Attribution CC BY-NCSA 4.0 license (https://creativecommons.org/licenses/by-ncsa/4.0/deed.es). 